\title{
Lateral flow test detects SARS-CoV-2 neutralising antibodies
}

Novodiax Inc., under the guidance of Dr Jianfu Wang, has produced a novel lateral thow detects neutralising antibodies (NAbs) against SARS-COV-2 (the Measuring NAbs is important to halp us determine whether vaccination programmes are effective by measuring the level of immunity of vaccine recipien They are also a vital tool for informing the strategies that will help us to achieve herd immunity from COVID-19. Lateral flow tests are convenient, versatile and relatively cheap. They crofessional healthced by a or by an individual at home. Lateral flow tests encourage mass testing and help to protect individuals from infection, reinfection, of COVID-19.

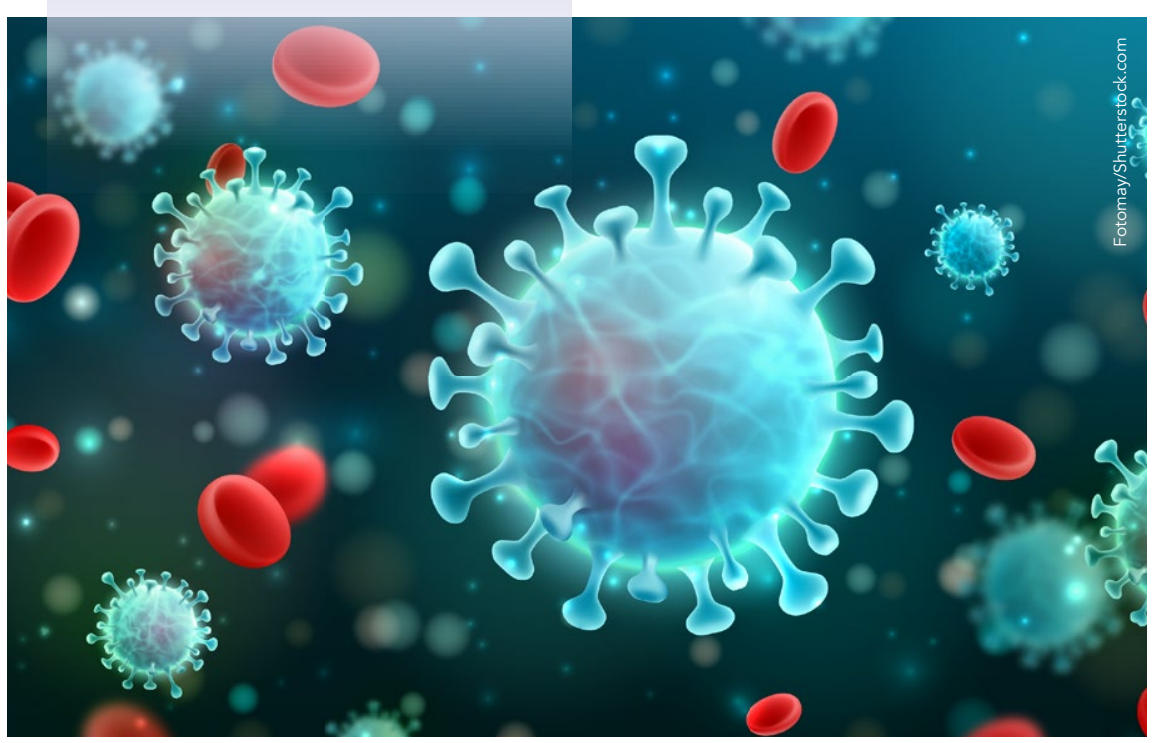

Specifically, NAbs block the interaction between the receptor-binding doma (RBD) of the spike protein of the SARS-CoV-2 virus and its receptor on the host cell, human angiotensinNAbs bind to the ACE2 bining site on the RBD, the spike protein cannot fuse to the host membrane or invade the cell. Thus, NAbs eliminate the infectious virus particle before any infection can occur. In contrast, the majority of non-neutralising BAbs bind specifically to the pathogen but do not interfere with its infectivity. Their role is to flag the invading pathogen for other immune cells to destroy.

\section{WHY IS A NAb LATERAL} FLOW TEST NEEDED? At the time of writing, there have 2.65 molle than 120 million cases and 2.65 million deaths from COVD-19 globally. Governments across the programmes and biotechnology companies are mass producing vaccines. This needs to be achieved as quickly as possible, so that communities can obtain a protective level of neutralising antibodies, or 'herd immunity'. This is important to kick start the economy and return to a semblance of normality after a year of battle against this novel coronavirus.

At present, there are many vaccines under development, with more than 30 in various stages of clinical trials. Almost all vaccines contain the virus' spike protein, so can induce NAb hostivation to block virus entry into to control the spread of the virus, a

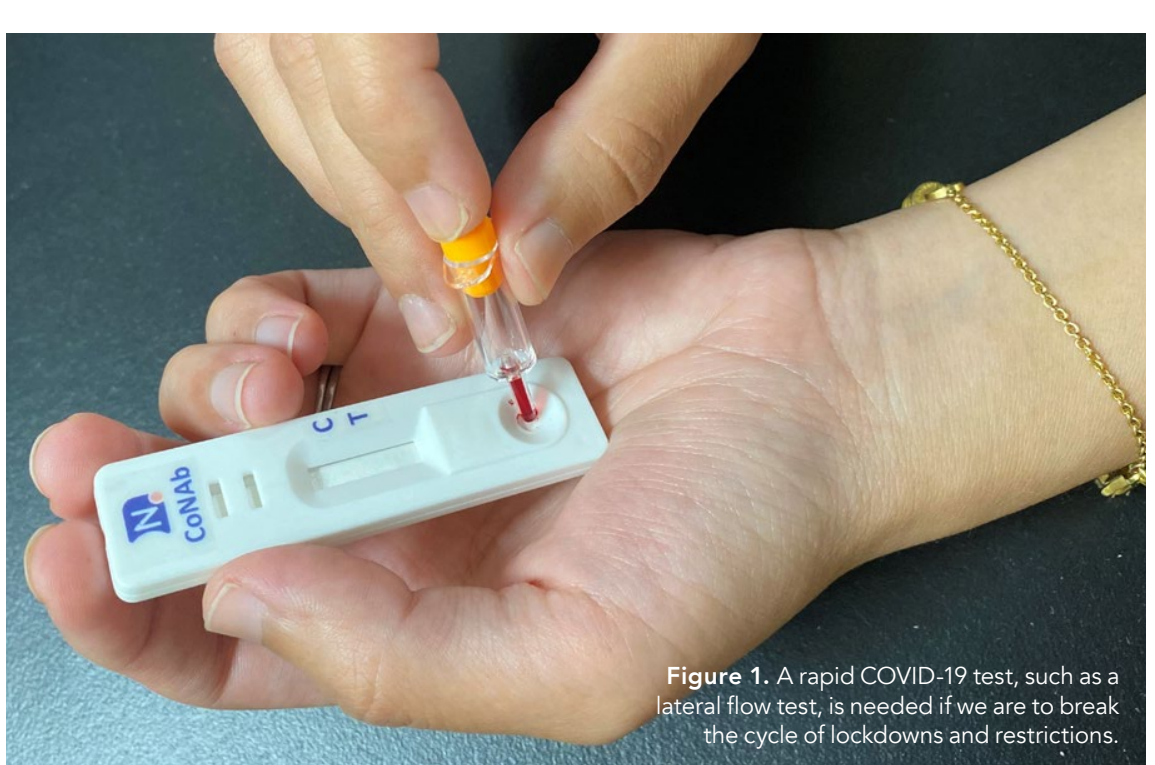

rapid test, such as a lateral flow test, is needed if we are to break the cycle levels are closely correlated with vaccin effectiveness and have been used as the primary laboratory measurement onset of symptoms and remain elevated is still not definitivance. However, the biological a tibody responses wir the biogch and protection from reinfection. The duration

Testing for NAb levels is therefore essential to manage the spread of the discase is to identin ind duals who f measuring NAb levels can ensure regular testing within communities. This data could be used by government officials and policymakers to determine the timeline of lifting lockdown restrictions, to more accurately anticipate the course of the pandemic, and to better understand our immunology against this novel coronavirus, aiding the development of future strategies. It can also inform researchers about the role NAbs play in eliminating the virus including the longevity of the protection they offer - and can protect communities from reinfection. In addition, the data ca be used to monitor levels of protective individuals.

\section{CONAB BY NOVODIAX}

Novodiax Inc. was founded in Californi mission to advance mmunoassays through innovation.

during vaccine $\quad$ Novodiax, under Such tests that Testing NAb levels is vital for managing lead researcher, $\mathrm{Dr}$ can continuously the spread of the coronavirus and to Jianfu Wang, has monitor NAb levels are required to
identify individuals who have to isolate. novel lateral flow determine the large-

scale success and longitudinal efficacies of of antibody responses required for vaccination programmes, and to prevent the spread of further infections.

ACHIEVING PROTECTION FROM SARS-CoV-2

Although multiple vaccines to the SARSCov-2 vins have been developed and protection to $100 \%$ of its recipients. For example, the protection rate of Pfizer-BioNTech's vaccine is 95\%, while Astrazencents is the development of a protective leve of NAbs that promises some defence against infection or reinfection. By mass-measuring vaccinated recipients' NAb levels, those who are not protected by the vaccines can be identified and revaccinated with a different vaccine or booster to achieve herd immunity.

There are still many unknowns when it comes to solving the puzzle of this novel coronavirus. For example, it has been predicted that antibody responses for

the virus can be detected in a majority of
infected individuals $10-15$ days after the is also not yet known. Levels of NAbs equipment to provide rapid results. communities to reach herd immunity produced varies greatly between patie and developed IgG antibodies (the main type of antibody) in patients with mild symptoms have a rapid half-life of
approximately 36 days. Reinfection cases as to the level of immunity that can be as to the level
developed.

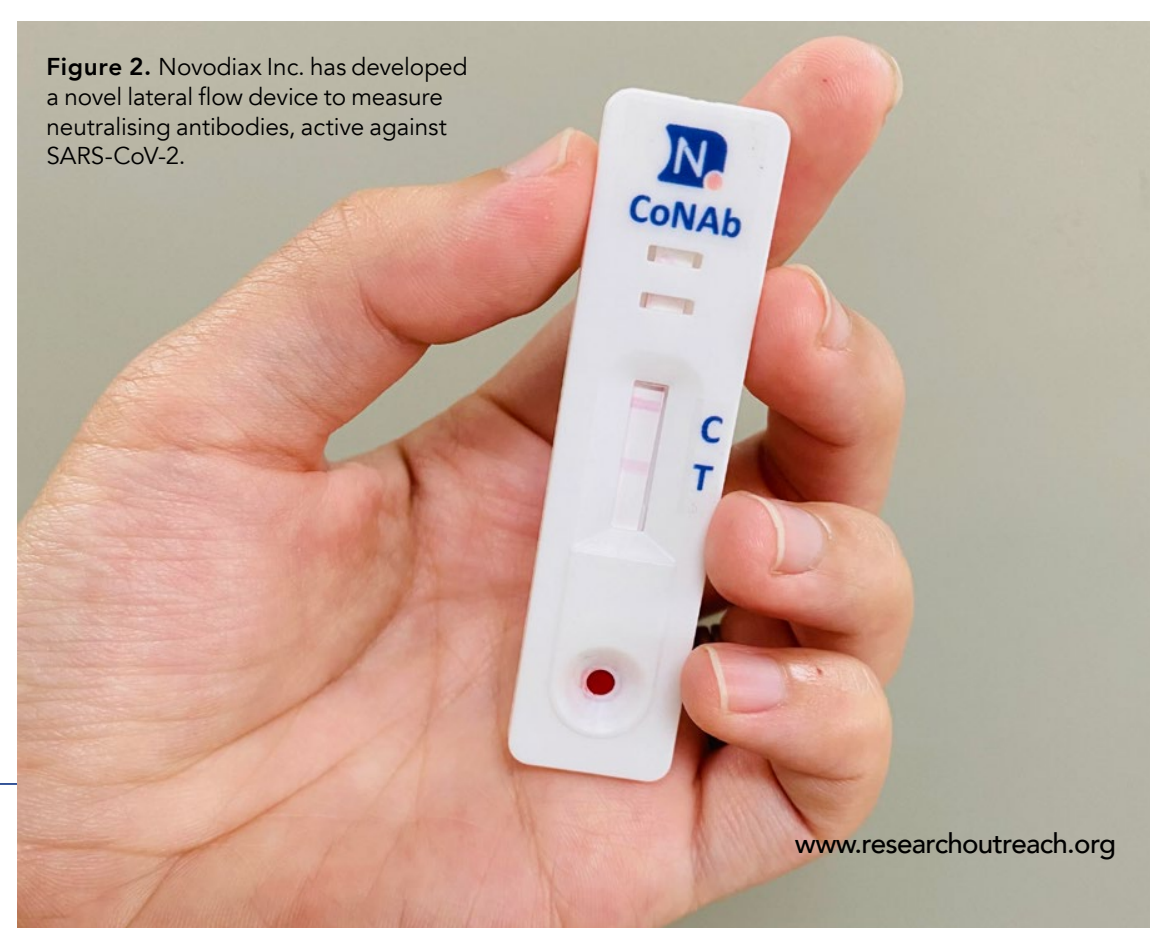

measure NAbs which are active against SARS-CoV-2, called CoNAb. LFDs are versatile, relatively inexpensive, and do not require laboratories with specialist They also only take mintes to obtain results, compared to other methods, such as ELISA tests, which may take FD works by detecting viral protein (entigens) from a sample. If there are 


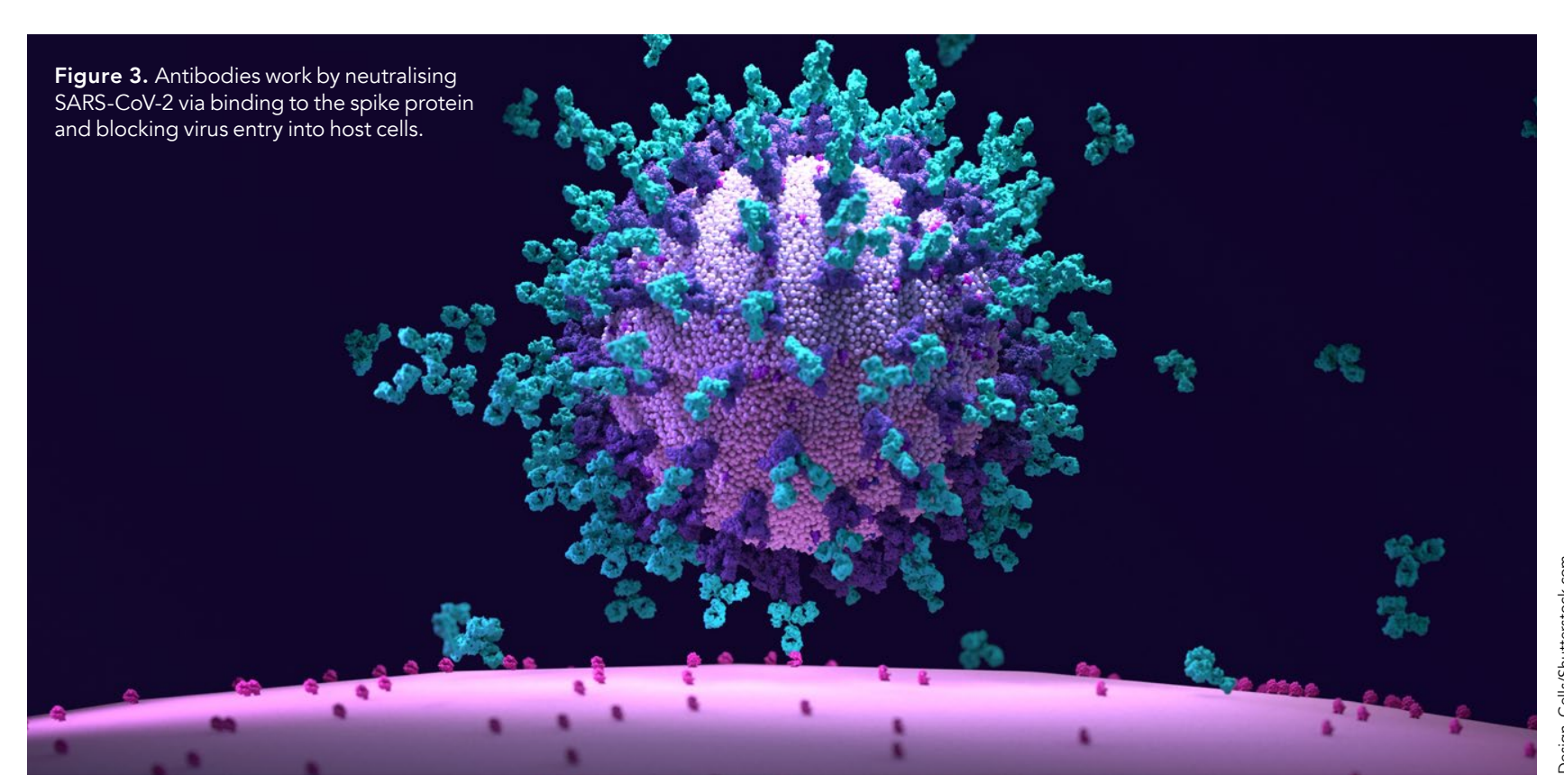

enough target antigens in the sample, visual signal will be generated.

The rapid NAb test kit works by testing for the inhibition of the spike protein of SARS-CoV-2. Gold nanoparticle (GNP) is bound to the RBD of the spike protein while ACE2 (the target receptor on the host cell) is embedded onto a specialised nitrocellulose membrane on the test strip. Like all lateral flow tests, approximately $10 \mu$ l of blood is taken from the patient and transferred onto the sample pad on the LFT device (Fig 1). 50 - 70pl of bufter is added to drive the blood sample through the device. Any NAbs present in the blood sample will neutralise or block the interaction between the RBD and ACE2. INAbs are not present (e.g., the person being RBD and ACE2 will interact and the gold nanoparticles will be detected producing a visual signal. However, if NAbs are present, then any GNP signal will be diminished, or will disappear.

At present, there are various methods to determine an individual's NAb levels. Firstly, conventional virus neutralisation tests are conducted in a biosafety leve three laboratory. These tests use live ACE2-expressing cells and SARS-CoV-2 or pseudovirus, and take $3-14$ days for results. Although the gold standard for NAb measurement, his method is labour mensive and requires specialst laboratoy workng condilons. A simpler assay (ELISA) has also been developed.
The EUSA mimics the ACE2-RBD (on the The ELISA mimics the ACE2-RBD (on the spike protein) interaction and detects
the amount of SARS-CoV-2 NAbs in few hours However the EUSA must be two laboratory. In contrast, the CoNAb measurement of NAb levels in individuals
is needed to inform policymakers and

Even though individuals are vaccinated, complete immunity against the virus.

minutes to complete. It is also the most convenient, as it can either be perform

\section{A RAPID AND ACCURATE TEST}

The Novodiax team compared the

efficacy and effectiveness of the CoNA LFD against conventional methods such tests and obtained highly comparable results as indicated in the references cited. Blood plasma samples were obtained from a Bio-Bank company and an in-house collection: 50 were negative, and 80 were normal plasma establish negative baselines. There was very high agreement $(91.14 \%)$ with cond $99 \%$ specifict for SARS bitito and convenient LFD test developed

epidemiology of the virus. The rapid effectiveness of COVID-19 vaccine. It could also provide a basis for immunity passports for individuals (rather than those who have ust received the vaccine).

A broad collaborative effort is now required to up-scale and optimise this product, so it can be utilised widely across different communities. who have developed SARSconducted in a specialist biosafety level it should not be assumed that they have PCR positive for SARS-CoV-2, 30 were obtained between 2014 and 2016 RT-PCR, 72.92\% sensitivity (the ability to
In conclusion, herd immunity against though individuals ar from a reality. Even should not be assumed that complete immunity against the virus. The is needed to inform policymakers an

\section{Behind the Research}

Dr Jianfu Wang

E: jianfuw@novodiax.com T: +1 (510) 3589609 W: www.novodiax.com 圈 YV4h8gmhxf0

Research Objectives

Novodiax Inc. have developed a lateral flow test to detect SARS-CoV-2 neutralising antibodies.

\section{Detail}

Address

3517 Breakwater Ave., Hayward, CA 94545, USA

Bio

Novodiax Inc. was founded in 2009 in California with mission to advance immunoassays through innovation. Novodiax Inc offers a 10-min immunohistochemistry product line worldwide, enabling oncology surgeons and surgical pathologists to make accurate diagnoses during surgery. Development of this rapid SARS-CoV-2 neutralising antibody test is a natural expansion of their original products.

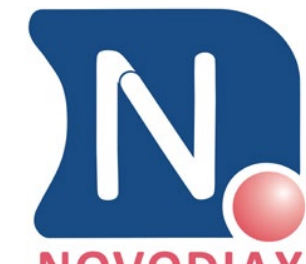

NOVODIAX

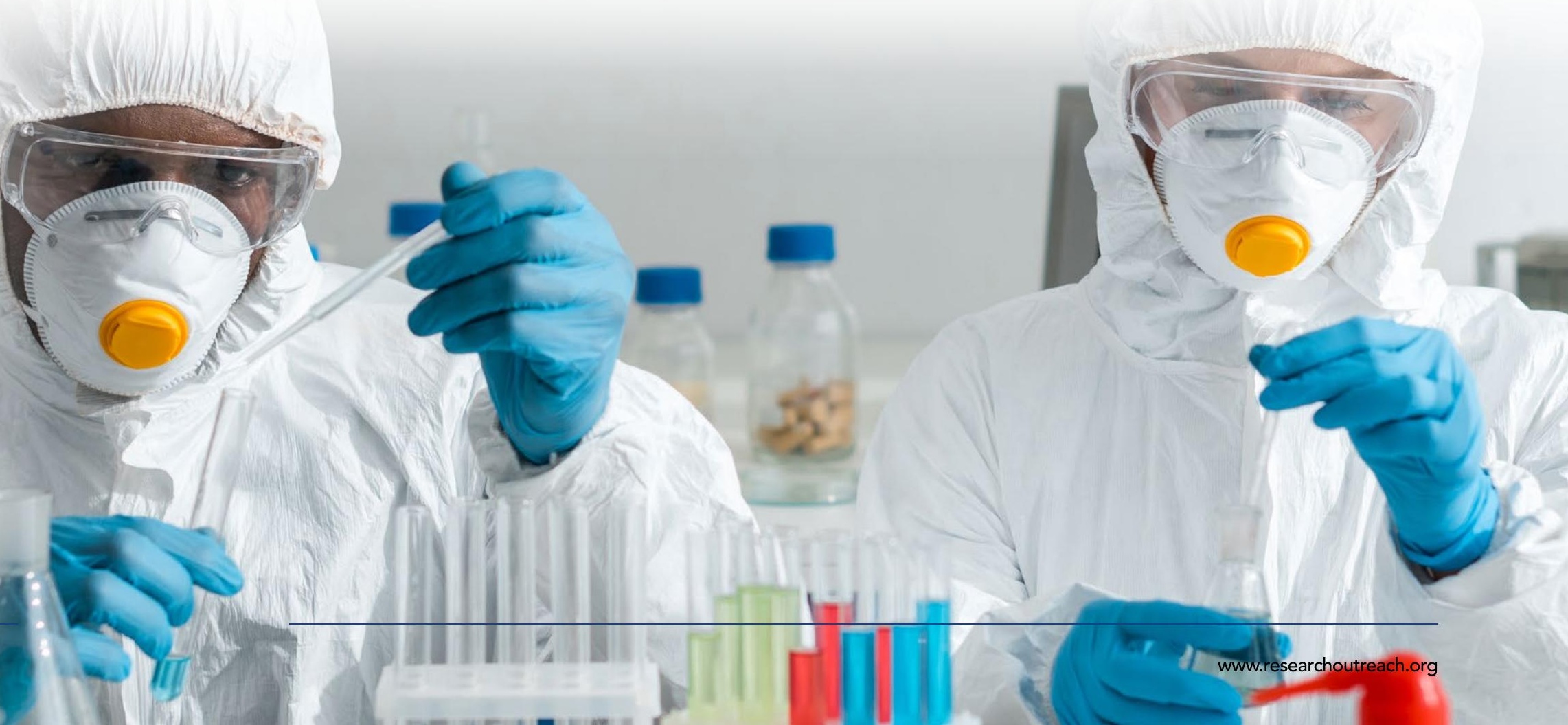

\section{References}

Mina, M. J., Peto, T. E., García-Fiñana, M., Semple, M. G. et al. (2021). Clarifying the evidence on SARS-CoV-2 antigen rapid tests in public health responses to COVID-19. The Lancet, [online]. https://www.thelancet.com/journals/lancet/article/ PIIS0140-6736(21)00425-6/fulltex

Zhang, N., Chen, S., Wu, J. V., Yang, X. et al. (2020). A lateral flow test detecting SARS-CoV-2 neutralizing antibodies. medRxiv, lonline.. hitps./Mww.medxiv.org/

\section{Personal Response}

How long will it take until the test is ready for general use?

II Novodiax is currently preparing applications for U.S. Emergency Use Authorization, and for equivalent regulatory requirements in other countries. Meanwhile, we mare ordering. We anticipate that a a product for general use will
be released around June 2021 . 